\title{
Análise de Impacto Ambiental em Unidades Funcionais de um Edifício Universitário: Uma Abordagem Piso por Piso
}

\section{Analysis of Environmental Impact on Functional Units as a University Building: A Floor-by-Floor Approach}

\begin{abstract}
${ }^{1}$ João Pedro Panagassi Forte, ${ }^{2}$ Vasco Fernando Ferreira Rato
${ }^{1}$ Mestre em Estudos do Ambiente e da Sustentabilidade, Instituto Universitário de Lisboa, (jota_panagassi@hotmail.com) ${ }^{2}$ Doutor em Engenharia Civil - Reabilitação do Património Edificado, Professor Associado do Instituto Universitário de Lisboa, Instituto Universitário de Lisboa, Av. das Forças Armadas, 1649-026 Lisboa, Portugal.

(vasco.rato@iscte.pt)

RESUMO: As universidades podem ser fontes geradoras de impacto ambiental em diferentes campos. Elas podem impactar tanto positivamente, quanto negativamente. Buscar entender como estes impactos acontecem e buscar uma forma de mensura-los é um importante caminho a perseguir em busca do desenvolvimento sustentável no âmbito acadêmico. Sendo assim, este artigo busca apresentar métodos e resultados de uma avaliação qualitativa do impacto ambiental e social de um conjunto de unidades funcionais de um edifício universitário piso por piso, considerando impactos negativos como, por exemplo, os originados pelo uso de energia, uso de água e recursos, bem como a geração de resíduos, mas também impactos positivos, como ensino, aprendizagem e pesquisa. A avaliação metodológica é pautada na análise de quatro diferentes pisos e uma extensão do último piso do edifício da Ala Autónoma, situada no Instituto Universitário de Lisboa - ISCTE/IUL. Neste contexto, o trabalho visa compreender melhor a relação entre a classificação atribuída para cada Impacto Ambiental e qual a sua influência nos diferentes espaços da área de estudo, buscando compreender como ocorre sua variação ao comparar a situação piso por piso. De acordo com a avaliação realizada, percebeu-se uma heterogeneidade dos espaços que são considerados mais impactantes para cada piso. Essa situação ocorre tanto para o tipo de espaço, quanto na discrepância da pontuação.
\end{abstract}

Palavras Chave: Avaliação de impacto ambiental, edifício, universidade, sustentabilidade.

ABSTRACT: Universities can be sources that generate environmental impact in different fields. They can impact both positively and negatively. Seeking to understand how these impacts happen and looking for a way to measure them is an important path to pursue in search of sustainable development in the academic field. Therefore, this article seeks to present methods and results of a qualitative assessment of the social and environmental impact of a set of functional units in a university building floor by floor, considering negative impacts such as those caused by the use of energy, use of water and resources, as well as the generation of waste, but also positive impacts, such as teaching, learning and research. The methodological assessment is based on the analysis of four different floors and an extension of the top floor of the Ala Autónoma building, located at the University Institute of Lisbon ISCTE / IUL. In this context, the work aims to better understand the relationship between the classification attributed to each Environmental Impact and what is its influence in the different spaces of the study area, seeking to understand how its variation occurs when comparing the situation floor by floor. According to the evaluation carried out, a heterogeneity of spaces was noticed, which are considered more impactful for each floor. This situation occurs both for the type of space and the discrepancy in the score.

Keywords: Environmental impact assessment, building, university, sustainability.

\section{INTRODUÇÃ̃O}

A universidade assume importante papel na formação de cidadãos críticos, instrumentados para a implementação dessas necessárias mudanças. Como promotora do desenvolvimento de novas tecnologias pode, ainda, estabelecer diretrizes e incentivar pesquisas sobre os diversos aspectos do controle da poluição relacionada com diferentes campos ambientais. Além disso, a Universidade desempenha importante papel como divulgadora e 
estimuladora de novas ideias, convidando a população a se empenhar na busca de soluções para a problemática ambiental (LEMOS, 2018).

Segundo a Associação Brasileira de Normas Técnicas (2015), a gestão ambiental é definida como parte do sistema de gestão que compreende a estrutura organizacional, as responsabilidades, práticas, procedimentos, processos e recursos para formulação, aplicação, revisão e manutenção da política ambiental pública ou empresarial. Portanto, um Sistema de Gestão Ambiental (SGA) é parte do sistema global de gestão de uma organização por meio do qual esta controla os seus aspectos ambientais, ou seja, as atividades, produtos e processos que provocam, ou podem vir a provocar, impactos ambientais.

A universidade necessita, portanto, assumir um papel de grande importância quando analisado a questão ambiental e social e seus diferentes aspectos. As Instituições de Ensino Superior (IES) necessitam buscar novos parâmetros como uma forma de gerenciar seus processos de maneira mais prática e eficiente, buscando se preocupar e se antever a possíveis problemas no futuro.

Sendo assim, as universidades, como qualquer outra instituição que funciona como uma prestadora de serviços, deveriam se preocupar com os impactos causados ao meio ambiente e à sociedade advindos de suas ações e das atividades de seus agentes e de todos aqueles que, de certa maneira, estão envolvidos com as atividades da instituição, sejam estas, direta ou indiretamente relacionada com a mesma (HASAN; MORRISON, 2011).

Uma maneira de medir e fornecer transparência aos esforços das universidades nesse sentido é por meio de relatórios e rankings de sustentabilidade. Esta avaliação e classificação, com base em critérios sustentáveis, serve de incentivo para análise mais específica dos aspectos em que ela precisa melhorar. Além disso, a comparação entre muitas universidades em todo o mundo pode resultar em ações bem-sucedidas sendo implementadas em diferentes países e, assim, obter melhor eficiência em termos de sustentabilidade (LEMOS, 2018).

No início dos anos 2000 existiam cerca de 140 Instituições de Ensino Superior que incorporavam alguma prática relacionada a políticas ambientais na administração e na gestão acadêmica. E atualmente, de acordo com o ranking da UI Green Metric da Universidade da Indonésia (2018), o número de universidades com programas de sustentabilidade no mundo todo já ultrapassam 700. Isso demonstra como um Sistema de Gestão Ambiental vem se tornando cada vez mais eficiente em ambiente acadêmico.

Assim, espera-se, que em um futuro próximo, um SGA universitário seja cada vez mais considerado um fator de necessidade para o gerenciamento da instituição e não mais um diferencial para a IES, buscando trabalhar com foco na ideia de melhoria contínua de seus processos internos e com equilíbrio ambiental.

\section{FUNDAMENTAÇÃO TEÓRICA}

O conceito de sustentabilidade em um campus é uma estratégia para a melhoria das práticas sustentáveis e para aumentar a conscientização entre alunos e funcionários sobre sustentabilidade e assuntos relacionados, como o termo impacto ambiental. Além disso, várias iniciativas são tomadas para implementar o conceito de sustentabilidade no nível universitário (ER; KARUDAN, 2016).

A Universidade também é considerada uma instituição com produção de impacto tanto de maneira positiva, quanto negativa. Por isso, em 2010, foi lançado pela Universidade da Indonésia o UI GreenMetric World University Ranking, que tem por objetivo fornecer o resultado da pesquisa online sobre as condições atuais e políticas relacionadas ao Campus Verde e Sustentabilidade nas Universidades ao redor do mundo (UNIVERSIDADE DA INDONÉSIA, 2019). 
Para analisar o impacto ambiental, é importante definir seus conceitos e termos. Principalmente ao se falar em impacto ambiental acadêmico, visto as diferentes áreas de pesquisa e atuação que uma IES pode fornecer. De acordo com o Ministério do Meio Ambiente (1986), a resolução Conama $\mathrm{n}^{\mathrm{o}} 1$ afirma que o impacto ambiental pode ser definido como:

"Qualquer alteração das propriedades físicas, químicas e biológicas do meio ambiente, causada por qualquer forma de matéria ou energia resultante das atividades humanas que, direta ou indiretamente, afetam:

I - A saúde, a segurança e o bem-estar da população;

II - As atividades sociais e econômicas;

III - A biota;

IV - As condições estéticas e sanitárias do meio ambiente;

V - A qualidade dos recursos ambientais."

Do mesmo modo, ao se analisar o impacto social em um IES, é necessário levar em consideração aspectos relacionados a temática. Os impactos sociais representam uma temática muito diversa nos seus diferentes campos de atuação, dentre eles Administração, Engenharia, Sociologia. Podem também estar relacionados com setores como governo, empresas privadas, organizações não governamentais (ONGs), fundações, universidades, dentre outros (MAZZA; MADRUGA; MACULAN; STRECK; RODRIGUES, 2016).

De acordo com UI Green Metric (2019), os principais pontos para análise de impacto ambiental e social das universidades incluem a coleta de informações básicas sobre o tamanho da Universidade e seu perfil de zoneamento (urbano, suburbano ou rural) o grau de espaço verde, consumo de eletricidade devido à sua ligação com a pegada de carbono, além de outros aspectos importantes como transporte, uso de água, gestão de resíduos, configuração e infraestrutura, energia e mudança climática e educação e pesquisa. Após todas estas análises, também é importante perceber como a Universidade está respondendo ou lidando com as questões da sustentabilidade por meio de políticas, ações e comunicação.

Para Juliatto (2011), as Instituições de Ensino Superior (IES) se assemelham a pequenos núcleos urbanos, devido ao fato de sua organização, função, magnitude e distribuição que alcançam suas infraestruturas, abrangendo, desde sistemas de transporte, moradia, alimentação, lazer, trabalho e saúde, até a gestão dos resíduos por elas gerados serem semelhantes. Portanto, um sistema de gestão sustentável em meio acadêmico tornou-se extremamente essencial como forma de gerenciamento das atividades acima citadas ou de qualquer forma de impacto ambiental proveniente de trabalhos e tarefas secundárias a estas.

Com estes fatos, este trabalho objetiva demonstrar, dentro de características do SGA da universidade e na metodologia aplicada, como são classificados os espaços dentro de cada piso e como eles influenciam a classificação de impacto atribuída a cada piso da área de estudo.

\section{MATERIAIS E MÉTODOS}

Em primeiro lugar, foram estabelecidos fatores de análise para cada impacto levantado pelo Sistema de Gestão Ambiental (SGA) da Universidade, como mostra o Quadro 1.

Além disso, foram estabelecidos dois principais índices de análise: Impacto Ambiental e Social (IAS) e Impacto Final (IF). Após a coleta desses dados referente as áreas e a análise dos impactos do SGA, os IFs de todos os espaços foram comparados piso por piso e o espaço mais impactante de cada piso na Ala Autónoma foi encontrado.

Primeiramente, os dados foram coletados em conjunto com a Instituição em relação aos impactos que já eram utilizados pelo SGA da mesma. Após essa coleta, os mais relevantes para 
este estudo foram selecionados para a área de estudo. $\mathrm{O}$ Quadro 1 mostra os impactos levantados. Além destes, existe também o fator "Tipo", que pode ser considerado negativo $(\mathrm{N})$ ou positivo $(\mathrm{P})$.

Quadro 1 - Relação Entre Impactos e Tipos de Impacto

\begin{tabular}{|c|c|}
\hline IMPACTO & TIPO \\
\hline Uso de energia & $\mathrm{N}$ \\
\hline Consumo de água potável & $\mathrm{N}$ \\
\hline Uso de recursos e materiais & $\mathrm{N}$ \\
\hline Novas construções & $\mathrm{N}$ \\
\hline Condução de eventos internos e externos & $\mathrm{N}$ \\
\hline Gestão de resíduos & $\mathrm{N}$ \\
\hline Pesquisa & $\mathrm{P}$ \\
\hline Ensino-Aprendizado & $\mathrm{P}$ \\
\hline Atividades de extensão & $\mathrm{P}$ \\
\hline
\end{tabular}

Criou-se Fatores de Análise para os impactos selecionados, após isso, eles foram classificados de acordo com a sua intensidade. Ao final, sete Fatores de Análise (Tipo, Modo, Magnitude, Duração, Alcance, Efeito e Reversibilidade) foram utilizados e classificados em três diferentes graus de acordo com a intensidade do fator. Estas notas estabelecidas foram: 0,1 (menor intensidade); 0,3 (média intensidade); e 0,5 (maior intensidade).

É importante enfatizar que o fator "Tipo" não tem pontuação numérica; isto ocorre porque ele é classificado apenas de duas maneiras, positiva e negativa. Portanto, caso o impacto seja considerado positivo, sua pontuação será positiva, caso o impacto seja considerado negativo, a pontuação será negativa.

Essa escolha na forma como é descrita cada categoria de impacto ambiental encontra respaldo no aspecto legal através da resolução Conama $n^{\circ} 1$, de 23 de janeiro de 1986. Cabe ressaltar que os impactos sociais foram acrescidos da mesma classificação, de modo equalizar os pesos atribuídos para ambos. Assim, os fatores foram classificados de acordo com a seguinte definição:

Modo - Indica se o impacto é direto ou indireto. Impacto direto - resultante de uma simples relação de causa e efeito; impacto indireto - ocorre mediante uma reação secundária em relação à ação inicial, ou, em alguns casos, quando faz parte de uma reação em cadeia, levando a ocorrência de outras ações secundárias.

Magnitude - A magnitude pode ser definida como uma extensão do efeito que determinado tipo de ação realiza em determinada característica ambiental e social. A magnitude pode ser trabalhada em dois tipos de escalas: espacial e temporal. Sendo classificada como Grande, Média e Pequena.

Duração - Indica se o impacto em questão é temporário, permanente ou cíclico. Impacto temporário - acontece quando o efeito (impacto) tem duração determinada; impacto permanente - quando, uma vez executada a ação, o efeito não para de manifestar-se em um prazo temporal conhecido; impacto cíclico - quando o efeito da ação se manifesta em intervalos de tempo que podem ser determinados.

Alcance - Este parâmetro indica se o impacto é local, regional, nacional ou internacional. Impacto local - quando a ação afeta apenas o próprio sítio e suas imediações; impacto regional - quando o impacto pode ser sentido além dos arredores do sítio onde acontece a ação; impacto nacional ou internacional - quando o componente ambiental e social afetado 
tem relevante interesse nacional ou internacional, afetando mais de uma região específica ou mais de um país.

Efeito - Indica o prazo no qual o impacto analisado ocorre. Pode ser classificado de três maneiras diferentes: forma curta, de médio ou longo prazo. Primeiro, impacto curto - quando o impacto (efeito) ocorre praticamente no mesmo instante em que acontece a atividade de transformação, ou também chamada, causa; segundo, impacto médio - quando o impacto ocorre em médio prazo, contando a partir do instante em que se ocorre a atividade de transformação; terceiro, impacto de longo prazo - quando o impacto acontece em um horizonte a longo prazo, contando a partir do momento em que se dá a atividade de transformação.

Reversibilidade - Indica se o impacto em questão é reversível ou irreversível, de acordo com as seguintes definições: impacto reversível - quando o parâmetro ambiental afetado retorna às suas condições originais depois de terminada a ação; impacto irreversível - quando o parâmetro ambiental e social afetado não volta para as condições iniciais em um prazo previsível.

Quadro 2 - Tipos de Fatores e Suas Notas

\begin{tabular}{|c|c|c|}
\hline Fator & Fator de Análise & Nota \\
\hline \multirow{2}{*}{ Tipo } & Positivo & - \\
\hline & Negativo & - \\
\hline \multirow{2}{*}{ Modo } & Direto & 0,5 \\
\hline & Indireto & 0,1 \\
\hline \multirow{3}{*}{ Magnitude } & Pequena & 0,1 \\
\hline & Média & 0,3 \\
\hline & Grande & 0,5 \\
\hline \multirow{3}{*}{ Duração } & Temporária & 0,1 \\
\hline & Cíclica & 0,3 \\
\hline & Permanente & 0,5 \\
\hline \multirow{3}{*}{ Alcance } & Local & 0,1 \\
\hline & Regional & 0,3 \\
\hline & Nacional/Internacional & 0,5 \\
\hline \multirow{3}{*}{ Efeito } & Curto & 0,1 \\
\hline & Médio & 0,3 \\
\hline & Longo & 0,5 \\
\hline \multirow{2}{*}{ Reversibilidade } & Reversibilidade & 0,1 \\
\hline & Irreversibilidade & 0,5 \\
\hline
\end{tabular}

De acordo com Forte e Rato (2019), ao utilizar este tipo de metodologia, primeiro é necessário fazer a soma desses fatores e, assim, obter o primeiro índice trabalhado, o IAS de cada impacto. Em segundo, adicionar o IAS de todos os impactos de um determinado espaço e, assim, obter o IAS final do mesmo, como mostrado na equação (1).

Somar as notas dos fatores e, assim, obter o IA de cada impacto:

$$
\text { IAS (Impacto) }=\sum \text { Fatores }
$$


Após isso, é necessário realizar a soma dos IASs de todos os impactos e, assim, obter o IAS de cada espaço, como pode-se perceber na equação (2):

$$
\text { IAS (espaço) }=\sum \text { IAS (Impacto) }
$$

Forte e Rato (2019) ainda consideram que, após estas somas, será necessário multiplicar o IA do espaço por sua respectiva área. Esta operação pode ser entendida na equação (3). Dessa forma, será possível obter o Impacto Final (IF) de cada espaço.

$$
\mathrm{IF}=\mathrm{IAS} \times \text { Área }
$$

onde Área representa a área total de cada tipo de espaço $\left(\mathrm{m}^{2}\right)$.

Depois disso, é necessário comparar o Impacto Final de cada espaço e, finalmente, analisar qual o IF é o mais representativo na comparação de todos os espaços - piso-a-piso.

\section{1 Área de estudo}

Ala Autónoma (Figura 1) é um dos principais edifícios da Instituição, constituído por quatro pisos principais, um parque de estacionamento subterrâneo e um terraço. De acordo com as plantas do edifício, seus espaços são divididos em: zona técnica; instalações sanitárias; auditório; escritórios; sala de aula; sala de professores; refeitório; e laboratório.

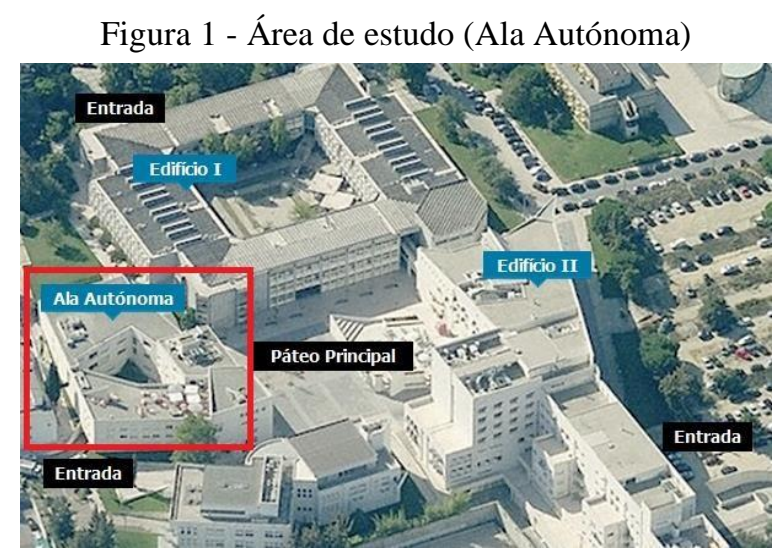

Fonte: ISCTE-IUL (2018).

Após uma análise das plantas foi possível perceber que os espaços estão distribuídos por 4 principais pisos da Ala Autónoma. Além destas plantas, ainda é possível encontrar no edifício um estacionamento subterrâneo (para efeitos de análise, foi considerado como Piso $\mathrm{O}$ ) e um piso superior denominado 4.1, por ser uma extensão do piso 4. Por meio destas plantas também foram encontradas as informações referentes as áreas e a disposição dos espaços piso a piso.

Um dos aspectos abordados neste artigo foi a comparação e definição de cada espaço de acordo com a classificação utilizada pela própria Universidade para definir a funcionalidade de cada um. Desse modo, uma análise mais próxima referente a realidade estrutural da Universidade foi elaborada, com uma análise piso a piso melhor definida.

Após a classificação dos espaços foi realizada a definição de suas respectivas áreas. No Quadro 3 tem-se os espaços (e suas respectivas siglas adotadas nas plantas da Universidade) e suas áreas analisadas piso por piso. 
Forte, João; Rato, Vasco; Análise de Impacto Ambiental em Unidades Funcionais de um Edifício Universitário: Uma Abordagem Piso por Piso. E\&S - Engineering and Science, 2020, 9:2.

Quadro 3 - Descrição dos Espaços e Áreas de Todos os Pisos

\begin{tabular}{|c|c|}
\hline \multicolumn{2}{|l|}{ Piso 0} \\
\hline Espaço & Área $\left(\mathbf{m}^{2}\right)$ \\
\hline Zona Técnica (ZT) & 60,6 \\
\hline \multicolumn{2}{|l|}{ Piso 1} \\
\hline Espaço & Área $\left(\mathbf{m}^{2}\right)$ \\
\hline Instalações Sanitárias & 58,4 \\
\hline Zona Técnica (ZT) & 62,9 \\
\hline Auditórios (AUD) & 250,7 \\
\hline Sala Comum (APO) (INV) (SRV) (GAB3) & 95 \\
\hline Sala de Aula (AUL) & 36,2 \\
\hline Gabinetes (GAB) & 241,6 \\
\hline Laboratório (GAB2) & 141 \\
\hline Restauração (CON) & 15,1 \\
\hline \multicolumn{2}{|l|}{ Piso 2} \\
\hline Espaço & Área $\left(\mathbf{m}^{2}\right)$ \\
\hline Instalações Sanitárias & 40,9 \\
\hline Sala Comum (APO) (INV) (SRV) (GAB3) & 278 \\
\hline Salas de Aula (AUL) & 354,4 \\
\hline Gabinetes (GAB) & 241,7 \\
\hline \multicolumn{2}{|l|}{ Piso 3} \\
\hline Espaço & Área $\left(\mathbf{m}^{2}\right)$ \\
\hline Instalação Sanitária & 40,9 \\
\hline Zona Técnica (ZT) & 7 \\
\hline Sala Comum (APO) (INV) (SRV) & 54,4 \\
\hline Salas de Aula (AUL) & 413,5 \\
\hline Gabinetes (GAB) & 241,6 \\
\hline Laboratório (GAB2) & 166,5 \\
\hline \multicolumn{2}{|l|}{ Piso 4} \\
\hline Espaço & Área $\left(\mathbf{m}^{2}\right)$ \\
\hline Instalação Sanitária & 19,4 \\
\hline Sala Comum (APO) & 191,5 \\
\hline \multicolumn{2}{|l|}{ Piso 4.1} \\
\hline Espaço & Área $\left(m^{2}\right)$ \\
\hline Zona Técnica (ZT) & 10,5 \\
\hline Sala Comum (SRV) & 82,4 \\
\hline
\end{tabular}




\section{RESULTADOS E DISCUSSÕES}

Percebe-se uma tendência maior do Impacto Ambiental ser negativo para este estudo, isso se deve pela maior quantidade de impactos que são classificados como negativos e devido a estes terem pesos mais relevantes em relação ao impactos classificados como positivos. Os impactos estudados podem ser vistos no Quadro 4.

Dentre os impactos que são classificados como positivos, suas pontuações são consideradas menos expressivas em comparação aos impactos negativos. Isso pode ser atribuído a um maior número de Fatores com pontuação mais baixa (Alcance, Magnitude e Duração), assim não conseguindo avaliações mais significativas para o estudo.

Esses Fatores, são classificados baseado em uma análise qualitativa, porém, sua interpretação em sua classificação são pautados em avaliações que tem como base definições na legislacão e, com isso, podem ser parametrizados de acordo com o que foi estipulado. Essa análise pautada nesse modelo de parametrização explica o motivo de alguns impactos possuírem valoração maior que outros tipos de impacto.

A área de estudo não estava a passar por nenhuma reforma estrutural em seus ambientes. Isto explica o fato do impacto "Construção nova e reabilitação" ter sido classificado com IAS igual a 0 .

Quadro 4 - Relação de Impactos e seus IAs

\begin{tabular}{|c|c|}
\hline IMPACTOS & IAS \\
\hline Utilização de energia & $-18,8$ \\
\hline Consumo de água potável & $-7,9$ \\
\hline Utilização de recursos materiais e produtos & $-14,4$ \\
\hline Construção nova e reabilitação & 0 \\
\hline Realização de eventos internos e externos & $-5,9$ \\
\hline Gestão de resíduos & $-7,8$ \\
\hline Investigação & 7 \\
\hline Ensino-aprendizagem & 9,4 \\
\hline Extensão universitária & 5,6 \\
\hline Total & $-32,8$ \\
\hline
\end{tabular}

Percebe-se neste quadro que, de acordo com o estudo, a Utilização de energia é considerada o impacto com valor mais representativo, com IAS de -18,8. Isto pode ser explicado pelo fato deste impacto estar presente em praticamente todos os espaços da Universidade. Como a sua fonte não é proveniente de fontes renováveis, sua classificação é atribuída valores maiores que os habituais. O que o torna um impacto de influência no resultado final dos espaços deste trabalho.

Outro impacto importante a se considerar é o fato da Utilização de recursos materiais e produtos também possuir IAS significativo. Este fato pode ser explicadopelo uso de produtos que são considerados difíceis de haver reciclagem ou diminuição da sua geração, como por exemplo: Isopores e plásticos. Isso causou um aumento na sua pontuação, principalmente relacionado ao fator Duração e Efeito, potencializando sua nota atribuída, além de ser presente em praticamente todos os espaços, com exceção dos espaços de uso periódico, como os Auditórios, pois o uso neste, depende do tipo de evento que está sendo realizado.

Contudo, para descobrir qual espaço por piso é mais impactante, é necessário realizar uma análise que leve em conta não somente o IAS de cada espaço, mas uma análise que torna 
importante ressaltar a dimensão espacial que cada sítio possuí. Assim tendo em conjunto dados como a área de cada espaço e os valores de IAS que foram supracitados, podemos analisar nos quadros 5, 6, 7, 8, 9 e 10 qual o Impacto Final (IF) atribuído a cada espaço e, após sua soma, qual o piso possuí maior valor agregado na área de estudo.

\subsection{Piso 0}

Piso 0 foi considerado um dos espaços menos relevantes para o estudo pelo fato de possuir apenas um espaço que, de acordo com a metodologia, não tem IF tão significativo em comparação com os outros espaços e, além disso, sua área se demonstra menor em comparação com outras áreas dos pisos subsequentes, como visto no Quadro 5.

Quadro 5 - Descrição dos Fatores e Notas

\begin{tabular}{|c|c|c|c|}
\hline \multicolumn{4}{|c|}{ PISO 0 } \\
\hline Espaço & Área $\left(\mathrm{m}^{2}\right)$ & IAS espaço & Impacto Final \\
\hline Zona Técnica (ZT) & 60,6 & $-6,3$ & $-381,78$ \\
\hline Total & 60,6 & - & $-381,78$ \\
\hline
\end{tabular}

Por meio desta análise é possível perceber que no Piso 0 o único espaço avaliado na metodologia proposta é a Zona Técnica. Este encontra-se presente no piso somente como espaço para armazenamento e utilização de produtos utilizados por funcionários.

\subsection{Piso 1}

O Piso 1, como visto no Quadro 6, é composto por 8 diferentes tipos de espaços, um dos pisos com maior heterogeneidade espacial no edifício, o segundo IF Total mais significativo do estudo. Neste piso, o fluxo de pessoas é o maior, isso pode ser constatado devido a heterogeniedade de espaço, visto que diferentes tipos de atividades podem ser realizadas nestes locais, tornando o piso atratativo e útil para diferentes nichos de pessoas. Essa heterogeniedade, tanto no tamanho das áreas, quanto nos valores de Impacto Ambiental e Social (IAS), causam uma variação ampla nos valores do Impacto Final (IF) também. Ness aspecto, pode-se perceber o quanto a área é significativa no valor do IF, mostrando o motivo pelo qual sua introdução nesta metodologia se torna necessária. No quadro 6 pode-se perceber como seus valores podem influenciar o resultado final de análise de Impacto Final do piso.

\begin{tabular}{|c|c|c|c|}
\hline \multicolumn{4}{|c|}{ Quadro 6 - Descrição dos Fatores e Notas } \\
\hline Espaço & Área $\left(\mathrm{m}^{2}\right)$ & IAS espaço & Impacto Final \\
\hline Zona Técnica & 62,9 & $-6,3$ & $-396,27$ \\
\hline Instalação Sanitária & 58,47 & $-8,8$ & $-513,92$ \\
\hline Auditório (AUD) & 250,7 & $-3,6$ & $-902,52$ \\
\hline Sala Comum (APO) (INV) (SRV) (GAB3) & 95 & $-5,8$ & -551 \\
\hline Sala de Aula (AUL) & 36,2 & $-3,1$ & $-112,22$ \\
\hline Gabinete (GAB) & 241,6 & $-0,5$ & $-120,8$ \\
\hline Laboratório (GAB2) & 141 & 0,2 & 28,2 \\
\hline Restauração (CON) & 15,1 & $-4,9$ & $-73,99$ \\
\hline Total & 900,9 & - & $-2.642,52$ \\
\hline
\end{tabular}


Um dos pontos a se perceber é que Laboratório é o único espaço com IA positivo. Isso deve-se ao fato de seus impactos ambientais serem predominantemente positivos. Um dos poucos espaços que foram classificados com os 3 impactos positivos levantados nesta pesquisa (Investigação, Ensino-Aprendizagem e Extensão Universitária). Devido a este espaço ser amplamento utilizado e tendo estes 3 impactos como predominantes, seu resultado de IA foram altos o suficiente para que sobresaissem aos impactos negativos, tornando, assim, este espaço com IF positivo.

Assim, é possível perceber que neste piso o espaço mais impactante é o Auditório. Isto deve-se ao fato dele possuir grande área que, em conjunto com seu IAS, faz com seu IF seja de $-902,52$, correspondendo a aproximadamente 34\% do Impacto Final do Piso 1. Um dos aspectos que faz seu IF de destacar em relação aos outros é sua área ser tão representativa neste piso, correspondendo a, aproximadamente $27,8 \%$ da área total do Piso 1 . Isso demonstra, como dito acima, o quão importante é o acréscimo da área como variável relevante a ser acrescida neste estudo.

Único espaço que possuí área semelhante a este espaço são os Gabinetes, com área de $241,6 \mathrm{~m}^{2}$. Porém, devido ao fato de seu IAS não ser tão significativo quanto o dos Auditórios resulta em um IF mais baixo de apenas -120,8, aproximadamente 7,5 vezes menor que IF dos auditórios.

Na Figura 2 tem-se a predisposição dos Auditórios neste andar, no qual pode-se perceber como sua área é relevante em comparação na análise por espaços neste piso.

Figura 2 - Auditório Piso 1

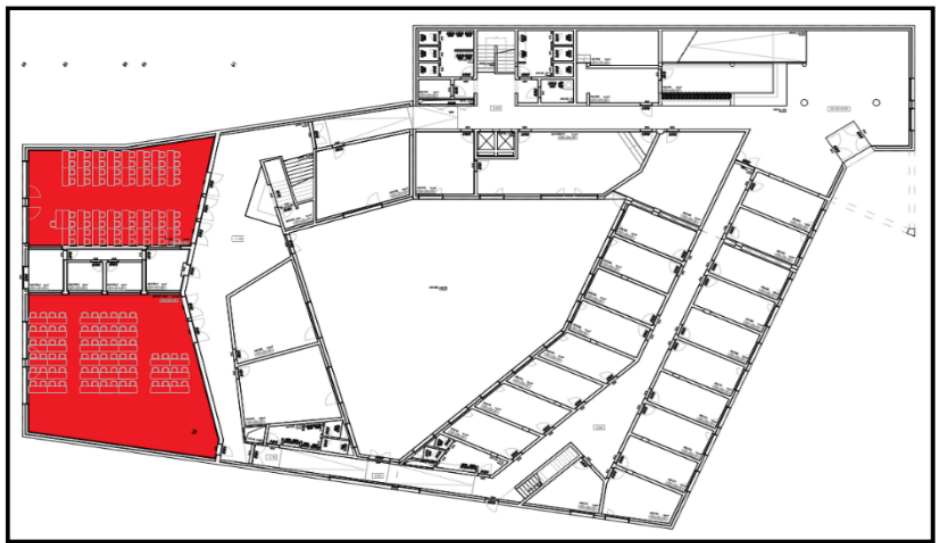

Fonte: ISCTE-IUL (2018).

Além disso, é necessário fazer uma observação relacionada as instalações sanitárias que, apesar de não possuírem grande área (aproximadamente 6,5\% da área estudada do piso e a menor entre os espaços) e não ser o espaço mais ambientalmente impactante no piso (segundo maior IF), ela possuí IAS relevante $(-8,8)$.

Pode-ser perceber que no piso 1 a interação entre Área e IAS é importante, sendo coodependentes para o resultado final do IF, alterando valores e trazendo mais variabilidade para o contexto de análise.

\subsection{Piso 2}

Este é um piso mais homogênio quando relacionado a variaedade de espaços, isso devese ao fato de ser considerado um ambiente voltado para a área administrativa e também para ministrar aulas. Outro ponto a se destacar é a ausência de espaços com avaliação positiva. $\mathrm{O}$ que faz com que os únicos espaços que existem, contribuirem para uma classificação negativa do piso, potencializando o efeito da avaliação dos IFs dos espaços na pontuação final. 
Percebe-se no Piso 2 que o espaço com maior valor de análise é a Sala Comum. Neste piso, apesar da pouco quantidade de espaços, contribuindo para a homogeniedade da área, os espaços com IF mais significativo e IF com menos significativos são bem definidos em comparação com o IF dos outros espaços.

Dentre todos os pisos, este é considerado o maior IF de todos os andares. Esse fator deve-se a uma junção de uma área total tão significativa quanto a do piso 1 e do piso 3, porém com IAS dos espaços maiores e suas respectivas áreas mais representativas.

Ao possuir mais homogeniedade na variedade de espaços e considerando o principal espaço (Sala Comum) em questão de representatividade quando analisa-se os valores de IAS e IF, percebe-se a razão que faz com o piso se torne aquele com maior IF. Assim, para este piso, possuir espaços homogênios é o fator principal que faz com que sua pontuação seja mais alta.

Quadro 7 - Descrição dos Fatores e Notas

\begin{tabular}{|c|c|c|c|}
\hline \multicolumn{4}{|c|}{ PISO 2} \\
\hline Espaço & Área $\left(\mathrm{m}^{2}\right)$ & IAS espaço & Impacto Final \\
\hline Instalações Sanitárias & 40,9 & $-8,8$ & $-359,92$ \\
\hline Sala Comum (APO) (INV) (SRV) (GAB3) & 278 & $-5,8$ & $-1.612,4$ \\
\hline Salas de Aula (AUL) & 354,4 & $-3,1$ & $-1.098,64$ \\
\hline Gabinetes (GAB) & 241,7 & $-0,5$ & $-120,85$ \\
\hline Total & 915 & & $-3.191,81$ \\
\hline
\end{tabular}

Com isso, constata-se que a Sala Comum corresponde a aproximadamente 50,5\% ($1612,4)$ do Impacto Final de todo o piso, mesmo possuíndo somente 30,3\% de toda área $\left(278 \mathrm{~m}^{2}\right)$, enquanto as salas de aula possuem $38.7 \%$ da área $\left(354,4 \mathrm{~m}^{2}\right)$, porém somente $34.42 \%$ do IF do piso $(-1098,64)$. Isso demonstra como um IAS maior influenciou mais no resultado do IF final, do que as suas áreas. Na figura 3 é possível perceber a distribuição das salas comuns no Piso 2.

Figura 3 - Sala Comum - Piso 2

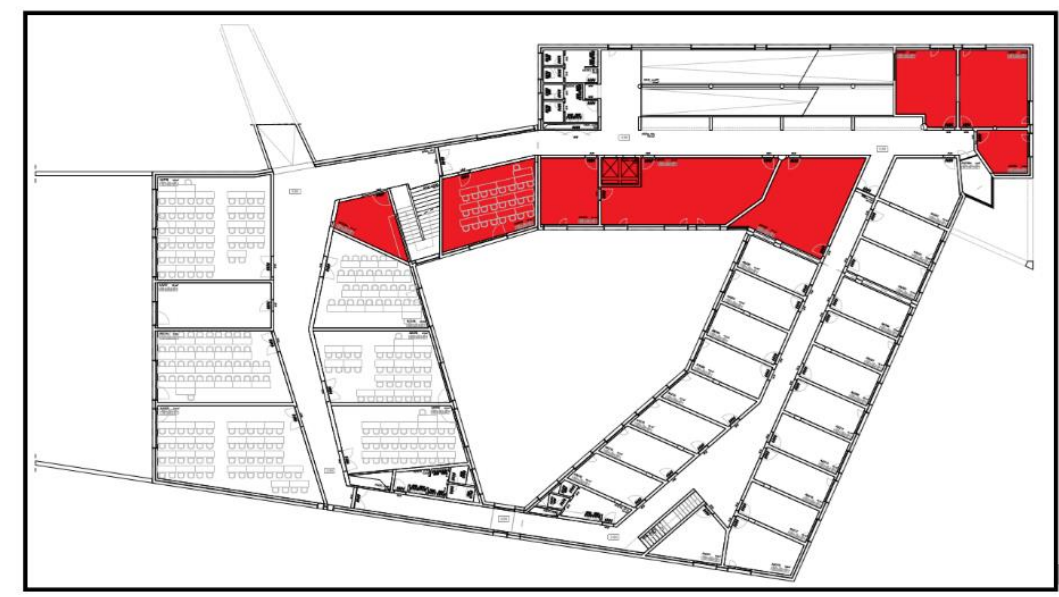

Fonte: ISCTE-IUL (2018).

No Piso 1, a área e o IA possuem relações diferentes no resultado do IF quando comparados com o Piso 2. Percebe-se, neste último, como o IA foi um ponto de desequilíbrio no resultado final, enquanto no primeiro piso a área foi um fator de desequlíbrio muito mais representativo. 
Forte, João; Rato, Vasco; Análise de Impacto Ambiental em Unidades Funcionais de um Edifício Universitário: Uma Abordagem Piso por Piso. E\&S - Engineering and Science, 2020, 9:2.

\subsection{Piso 3}

A análise mostra que no Piso 3 o espaço mais impactante é o das Salas de Aula. Neste piso, o espaço com IF significativo é bem definido e muito superior aos outros IFs, sendo considerado bem mais representativo. Isso pode ser percebido quando se analisa que o espaço corresponde a aproximadamente $61,3 \%$ do IF de todo o piso.

\begin{tabular}{|c|c|c|c|}
\hline \multicolumn{3}{|c|}{ PISO 3 } \\
\hline Espaço & Área $\left(\mathrm{m}^{2}\right)$ & IAS espaço & Impacto Final \\
\hline Zona Técnica & 7 & $-6,3$ & $-44,1$ \\
\hline Instalações Sanitárias & 40,9 & $-8,8$ & $-359,92$ \\
\hline Sala Comum (APO) (INV) (SRV) & 54,4 & $-5,8$ & $-315,52$ \\
\hline Salas de Aula (AUL) & 413,5 & $-3,1$ & $-1.281,85$ \\
\hline Gabinetes (GAB) & 241,6 & $-0,5$ & $-120,8$ \\
\hline Laboratório (GAB2) & 166,5 & 0,2 & 33,3 \\
\hline Total & 923,9 & & $-2.088,89$ \\
\hline
\end{tabular}

Este piso possuí enfoque voltado para atividades acadêmicas, por isso nota-se a predominancia da Sala de Aula com espaço mais representativo. Como fato importante, sua área é muito superior aos outros espaços, correspondendo a $44,7 \%$ da área total do piso do edifício, mesmo possuindo apenas o quarto maior IAS do piso. Sua área é mais significativa para o IF do resultado final do que o IAS, como pode ser visto no quadro 8 .

Neste caso, percebe-se como a Área foi a variável significativa no valor final de IF. Percebe-se que os pesos das variáveis no resultado final do IF varia de piso para piso demostrando que cada espaço possuí a sua particularidade neste estudo.

Apesar das Salas de Aula serem os espaço que mais se destaca neste piso, percebe-se que ele tem uma composição muito homogênea e com a classificação de IF considerada baixa se comparado os mesmos espaços, porém de outros pisos. Isso pode ser percebeido por dois fatores, uma menor área em alguns espaços, e um IAS baixo, o que faz com que seu IF seja baixo também. Esta condição pode ser percebeida em todos os espaços do piso, com excessão do mais impactante, as Salas Comuns.

Outro ponto é que a presença de um laboratório, sendo este considerado o único espaço com IF positivo, não diminuí de maneira significativo o IF do piso todo, pois todos os outros espaços tem IAS negativo e numericamente maior, dando muito mais peso para os espaços com mais impactos negativo que positivo.

A Figura 4 demonstra como este espaço é significativo em área (44,7\% do total do piso). Outro espaço que possuí área relativamente significante no piso são os Gabinetes $(25,9 \%$ do total do piso), porém como seu IAS é mais baixo, seu Impacto Final não é significativo. 
Forte, João; Rato, Vasco; Análise de Impacto Ambiental em Unidades Funcionais de um Edifício Universitário: Uma Abordagem Piso por Piso. E\&S - Engineering and Science, 2020, 9:2.

Figura 4 - Salas de Aula - Piso 3

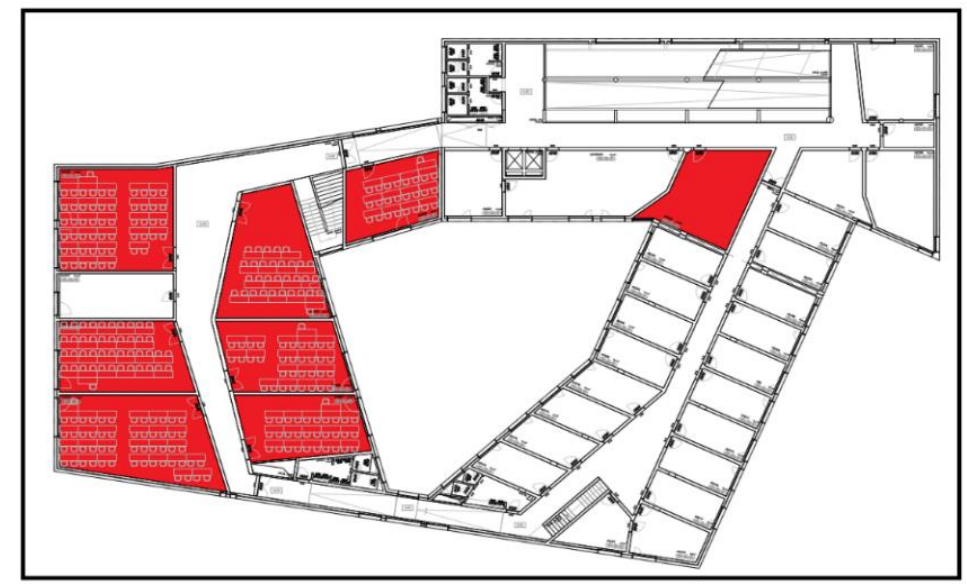

Fonte: ISCTE-IUL (2018).

\subsection{Piso 4}

É possível perceber que no Piso 4 o espaço mais impactante é a Sala Comum, sendo o IF mais significativo dos doi espaços. Isso se dá pelo fato deste piso possuir apenas dois espaços, sendo Sala Comum aquele com valor mais significativo representando 86,6\% de todo o Impacto Final do Piso 4.

Quadro 9 - Descrição dos Fatores e Notas

\begin{tabular}{|c|c|c|c|}
\hline \multicolumn{5}{|c|}{ PISO 4} \\
\hline Espaço & Área $\left(\mathrm{m}^{2}\right)$ & IAS espaço & Impacto Final \\
\hline Instalação Sanitária & 19,4 & $-8,8$ & $-170,72$ \\
\hline Sala Comum (APO) & 191,5 & $-5,8$ & $-1.110,7$ \\
\hline Total & 210,9 & - & $-1.281,42$ \\
\hline
\end{tabular}

Outro fato importante é que a área deste espaço representa 90,8\% da área total, sendo o ponto de desequilíbrio da análise na obtenção do IF deste piso, como pode ser visto na Figura 5. Este piso funciona em conjunto com o Piso 4.1, sendo ambos utilizados frequentemente para a realização de eventos que envolvam atividades da Ala Autónoma.

Figura 5 - Sala Comum - Piso 4

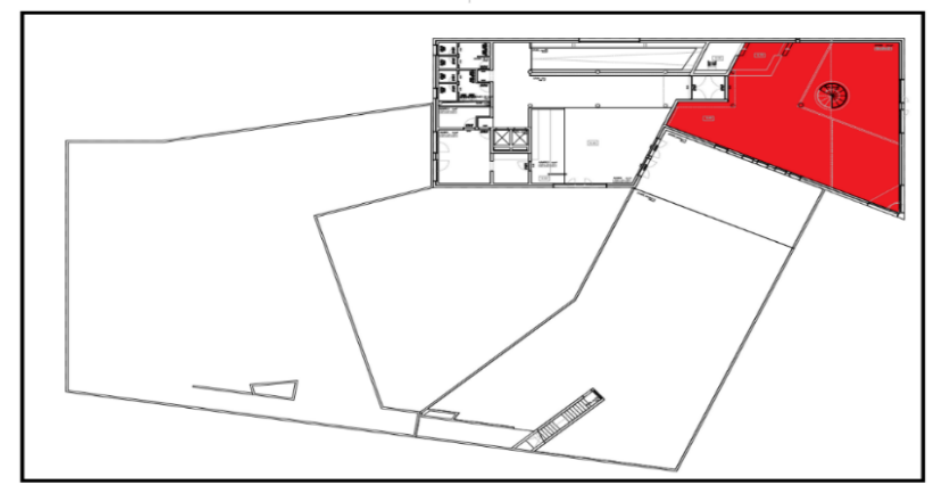

Fonte: ISCTE-IUL (2018).

Portanto, com uma área pequena, pouca variabilidade de espaços e com IAS baixo, faz com que o IF desse piso seja bem menor em relação aos outros. Isso não significa que ele seja, necessariamente, menos impactante que os outros pisos, mas sim, que sua relação área e IAS 
resulta em um valor numérico mais próximo de 0 do que com outros pisos, mas, ainda sim, negativo. O que torna este piso e todos os outros pisos, negativamente impactante.

\subsection{Piso 4.1}

O último Piso, o 4.1, pode ser considerado uma extensão do Piso 4, sendo considerado como área para atividades de lazer no edifício, como dito acima. Este andar possuí uma área pequena, com a existência de poucos espaços, sendo estes uma zona técnica e uma sala comum.

\begin{tabular}{|c|c|c|c|}
\hline \multicolumn{4}{|c|}{ Quadro 10 - Descrição dos fatores e notas } \\
\hline PISO 4.1 \\
\hline Espaço & Área $\left(\mathrm{m}^{2}\right)$ & IAS espaço & Impacto Final \\
\hline Zona Técnica (ZT) & 10,5 & $-6,3$ & $-66,15$ \\
\hline Sala Comum (SRV) & 82,4 & $-5,8$ & $-477,92$ \\
\hline Total & 92,9 & - & $-544,07$ \\
\hline
\end{tabular}

O IF do Piso 4.1 só não é menos significativo que do Piso 0 e, consequentemente, muito menor do que os IFs dos outros pisos. É um sítio pouco utilizado e, portanto, devido a sua área e a pouco variação de espaços no local, fez com que seu IF fosse bem inferior em comparação aos outros pisos. Se comparado com o Piso 2, que possui o maior IF dos pisos analisados, ele é aproximadamente 6 vezes menor.

Percebe-se que os fatores Área e IAS são pontos que precisam atuar de maneira conjunta para que o Impacto Final seja considerado alto ou baixo. Para que o IF do piso seja signifcativo, é necessário uma maior homogeniedade dos espaços, porém essa homogeniedade necessita estar alinhada com uma área significativa e um IAS minimamente relevante. Como visto, por exemplo, no piso 2, em que este possuí dois espaços com IF significativo, além de uma baixa diversidade de espaços. Esta afirmação pode ser valida tanto para análise de espaços e de pisos, como também, para ambientes considerados positivamente ou negativamente impactantes. Como nos Laboratórios, que é o único espaço avaliado como positivo, porém como sua área e seu IAS nos pisos não demonstram valor significativo e ponto de interferir de maneira significativa no estudo.

Ao analisar os pisos, percebe-se que todos eles são negativamente impactantes. Mostrando que os impactos positivos, além de estarem em menor quantidade, também são impactos que demonstam pouca força em detrimento dos impactos negativos. Tanto em valor, quanto em incidência.

As Salas Comuns no Piso 2 são os espaços mais impactantes dentre todos os espaços levantados em todos os pisos. Mesmo não possuindo o maior IAS ou a maior área dentre todos, este espaço possuí o maior equlíbrio entre as duas variáveis. Portanto, isso corrobora com o fato dessas variáveis serem complementares para a classificação de um IF alto ou baixo.

\section{CONCLUSÃO}

Ao final da realização deste trabalho, diferentes conclusões podem ser tomadas, algumas podem ser consideradas como óbvias, porém outras podem ser consideradas mais complexas.

Além das variáveis levantadas como o Impacto Ambiental e Social (IAS) e Área dos espaços estudados, outras variáveis poderiam ser incluídas na análise, como por exemplo fluxo de pessoas, consumos de recursos em diferentes épocas do ano, entre outros. Portanto, outros estudos com a acréscimo dessas variáveis se tornam imprescindíveis para um resultado cada vez mais fidedigno a realidade. 
Percebe-se que em todos os pisos, de acordo com a metodologia analisada, podem ser considerados ambientalmente impactantes, pois todos os pisos possuem IF negativo. Piso 0: 381,78, Piso 1: -2.642,52, Piso 2: -3.191,81, Piso 3: -2.088,89, Piso 4: $-1.281,42$ e Piso 4.1: 504,07. O fato de todos os pisos serem considerados negativamente impactantes deve-se ao IAS da maioria dos impactos ser negativo, restando somente Investigação, Ensino-aprendizagem e Extensão universitária como exemplos de impactos positivos nos pisos da Ala Autónoma. Portanto, para mudar este cenário é preciso buscar incentivar novos impactos positivos e minimizar os efeitos dos negativos.

Percebe-se como nos diferentes tipos de pisos, os espaços considerados mais impactantes são distintos. Ao considerar-se os 4 principais pisos do edifício, percebe-se como a distribuição do impacto na área do estudo é heterogênea, de acordo com a metodologia, sendo Auditórios (Piso 1), Sala Comum (Piso 2), Salas de Aula (Piso 3) e, novamente Sala Comum (Piso 4) os espaços com IF mais significativos.

É possível entender ao analisar os resultados, como a relação área e IAS são importantes, pois de maneira geral, o que faz com que o IF possua uma classificação significativa, sendo ela positiva ou negativa, é possuir uma área do espaço bem representativa. Assim, mostra que a área do espaço precisa e deve ser levada em consideração nestes tipos de estudo.

É preciso, estabelecer uma escala de análise para que se possa perceber como analisar um determinado grau de IF que um local tem para outro, tendo assim, uma base comparativa mais simplificada para os diferentes tipos de locais.

Espera-se que esta pesquisa possa ser trabalhada em diferentes tipos de ambientes, independentemente de seu tamanho ou sua função (empresa, universidade, cidades, entre outros).

Também é importante perceber que a significância atribuída a cada tipo de impacto em cada espaço distinto deve, ainda, ser analisada por meio da atribuição de diferenças de atratividade em modelos de análise multicritério dentro de sessões de grupos focais. Assim, será possível definir pesos que representem novos cenários de análise progressivamente mais completos.

\section{REFERÊNCIAS}

ASSOCIAÇÃO BRASILEIRA DE NORMAS TÉCNICAS. NBR ISO 14001: Sistema de Gestão Ambiental - elaboração. Rio de Janeiro, 2015. p. 8.

BRASIL. Ministério do Meio Ambiente. Resolução CONAMA nº1. Diretrizes Gerais para a avaliação de impacto ambiental. Brasil. Fonte: Ministério do Meio Ambiente. Brasília. 1986.

ER, A.C.; KARUDAN, R. Promoting campus sustainability: A conceptual framework for the assessment of campus sustainability. Journal UKM. Malasia v. 11, n. 2, p. 36-49, 2016.

FORTE, J.P.F.; RATO, V. Energy environmental impact of functional units of a university building. In: WORLD CLIMA CONGRESS, XIX., v.6. 2019, Bucareste. Anais: Bucareste, REHVA, 2019, p.1-8, 2019.

HASAN, M.; MORRISON, A. Current University Environmental Management Practices. Journal of Modern Accounting and Auditing. v. 11, n. 1, p. 2-5, 2011.

JULIATTO, C. J. Gestão Integrada de Resíduos Sólidos para instituições públicas de ensino superior. Revista Gual, v. 4, p. 170-193, 2011. 
LEMOS, P. L. I.; BRANDO, F. R.; ALMEIDA, P.; MULFARTH, R. C. K.; APRILANTI, T. M. G.; MARQUES, L. O. A.; JORGE, N. L.; MALHEIROS, T. F. The University of São Paulo on the 2017's GreenMetric Ranking. In: E3S Web of Conferences, n. 48, 2018, online. Anais: International Workshop on UI GreenMetric World University Rankings- IWGM. p. 6-8. 2018.

MAZZA, V. M. S.; MADRUGA, L. R. R. G.; MACULAN, C. G.; STRECK, L.; RODRIGUES, M. C. M.; Emergência da temática impacto social: uma análise da produção científica através da base web of Science. Revista do CEPE. Santa Cruz do Sul, n. 44, p. 52-67, 2016.

UI GREEN METRIC. UI GreenMetric World University Ranking - Methodology. Indonésia. Disponível em: http://greenmetric.ui.ac.id/methodology-new/\#15602199646216b9d5419-9e0a. Acesso em: 07 jul. 2020.

UNIVERSITAS INDONESIA. UI GreenMetric World University Ranking. Indonésia. Disponível em: http://greenmetric.ui.ac.id/what-is-greenmetric/. Acesso em: 08 jul. 2020. 